\title{
Novel OFDM System Using Orthogonal Pilot Symbols with Subcarrier Index Modulation
}

\author{
Y. ACAR and S. ALDIRMAZ ÇOLAK
}

\begin{abstract}
In this work, two new transmission schemes are proposed to increase the spectral efficiency of orthogonal frequency division multiplexing (OFDM). In practical OFDM systems, channel estimation is usually performed by employing pilot symbols which is based on inserting known symbols in the time-frequency domain. However, pilot symbol designing is one of the bottlenecks of OFDM systems limiting the increase of spectral efficiency. We apply new pilot design structures, which are use orthogonal Walsh-Hadamard codes. To increase the spectral efficiency of OFDM systems, we assign the extra bits to the index of the each orthogonal Walsh-Hadamard codes. Simulation and theoretical results show that proposed methods have better performance than conventional OFDM with higher spectral efficiency. Moreover, no more energy is spent for additional information carried in the indices compared to classical OFDM systems. As a result, proposed methods provide both spectral efficiency and energy efficiency.
\end{abstract}

Index Terms - Orthogonal frequency division multiplexing (OFDM), subcarrier index modulation (SIM), channel estimation, Walsh-Hadamard codes.

\section{INTRODUCTION}

$\mathrm{O}$ RTHOGONAL frequency division multiplexing (OFDM) has attracted a lot of attention for broadband wireless communication systems due to robustness against frequencyselective fading channels and simple implementation [1]. OFDM has been adopted by several popular standards such as long term evolution-advanced (LTE-A), worldwide interoperability for microwave access (WiMAX), local area network (LAN), and digital video broadcasting (DVB) [2-3]. The channel estimation is a crucial and challenging procedure for OFDM systems in practical scenarios especially for mentioned standards above. In [4], OFDM performance is presented for pedestrian and vehicular channel models, i.e., practical channels.

Channel estimation (CE) techniques can be roughly divided up into two groups, i.e., using blind-way or pilot symbol aided (PSA) [5, 6]. While blind channel estimation methods are based on second or higher order statistics; pilot-assisted

YUSUF ACAR, is with STM Savunma Teknolojileri Mühendislik ve Ticaret A.Ş., Ankara, Turkey, (e-mail: yusuf.acar@stm.com.tr).

(iD https://orcid.org/0000-0002-3956-1097

SULTAN ALDIRMAZ COLAK, is with Kocaeli University, Kocaeli Turkey, (e-mail:sultan.aldirmaz@kocaeli.edu.tr).

iD http://orcid.org/0000-0001-7154-0723

Manuscript received June 08, 2019; accepted September 16, 2019. DOI: $10.17694 /$ bajece.588919 channel estimation methods transmit a known signal, pilots, in both transmitter and receiver sides. Blind-way techniques have higher spectral efficiency compared to that of PSA; however their performance are poor due to the data interference. Moreover, their computational complexity is higher than that of PSA techniques. Several PSA-CE schemes for OFDM applications have been investigated $[7,8,9,10]$. Since pilot symbols are transmitted instead of data symbols, spectral efficiency of PSA-CE methods is lower than blind-way CE techniques. Interested readers are referred to [11] and the references therein for a review of OFDM channel estimation techniques.

Spatial modulation (SM), which has been recently proposed for multi-input multi-output (MIMO) systems, is a very distinct and useful modulation scheme [12]. Using the idea in $\mathrm{SM}$, it was demonstrated by sub-carrier index modulation (SIM) (i.e., OFDM-IM) that spectral efficiency could be increased for OFDM systems [13, 14, 15]. SIM concepts have attracted increasing attention as a promising technique for the next generation wireless communication systems in the last decade. SIM method allows to transmit additional bits to the conventional modulation schemes by mapping the data bits to the indices of the different medias. For more details on this important field, the interested researchers are referred to survey on SIM schemes in [16].

Recently, two blind CE techniques for OFDM systems have been proposed by inspired the SIM technique [17]. The positions of the pilot signals in the frequency domain of the OFDM signal (before IFFT operation) transmit additional data bits in addition to the modulated symbols. Thus, the spectral efficiency of the system is increased.

The Walsh-Hadamard matrix [18] or pseudo-codes are key components of modern information technology such as code division multiple access (CDMA) communication systems. Walsh-Hadamard matrices are square matrices whose entries are $\mathbf{- 1}$ or $\boldsymbol{+ 1}$, and columns or rows are orthogonal to each other. Assume that we have $N_{p} \times N_{p}$ Walsh-Hadamard matrix as follows:

$$
\mathbf{C}=\left[\begin{array}{cccc}
c_{1,1} & c_{1,2} & \cdots & c_{1, N_{p}} \\
c_{2,1} & c_{2,2} & \cdots & c_{2, N_{p}} \\
\vdots & \vdots & \ddots & \vdots \\
c_{N_{p}, 1} & c_{N_{p}, 2} & \cdots & c_{N_{p}, N_{p}}
\end{array}\right]
$$

where $N_{p}$ denotes number of pilot symbols. 


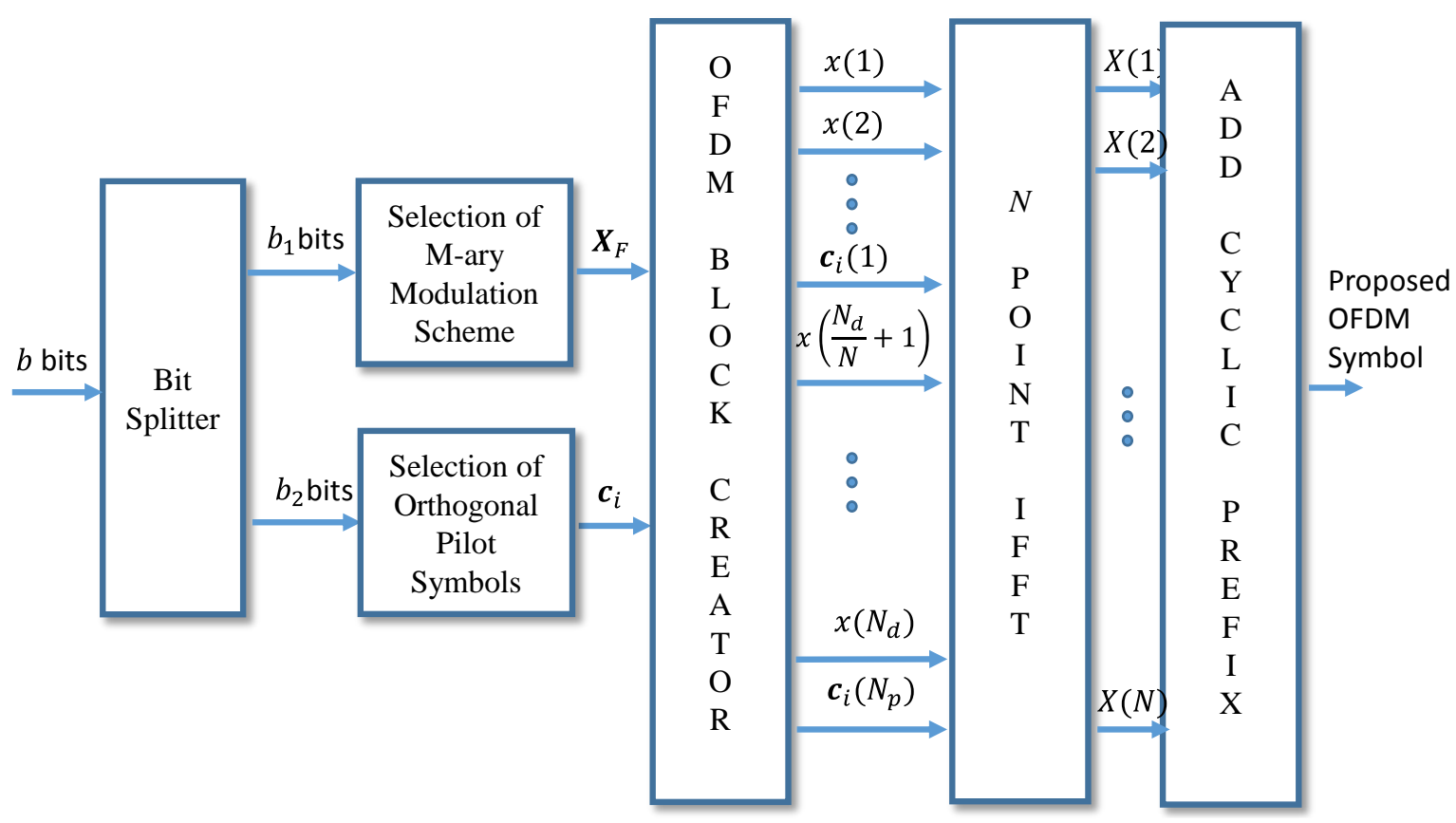

Fig.1. Block Diagram of the Proposed Methods

We can re-written Walsh-Hadamard matrix as $\mathbf{C}=\left[\begin{array}{llll}\mathbf{c}_{1} & \mathbf{c}_{2} & \cdots & \mathbf{c}_{N_{p}}\end{array}\right]^{T}$ where $\quad \mathbf{c}_{1} \mathbf{c}_{2} \quad \cdots \quad \mathbf{c}_{N_{p}} \quad$ are $\quad 1 \times N_{p}$ orthogonal vector. Inspired by SIM concept and orthogonality of Walsh-Hadamard codes, we proposed two novel channel estimation techniques to increase the spectral efficiency of OFDM systems. The basic idea of the proposed techniques is to select pilot symbol vectors based on the incoming bits from possible pilot vector candidates.

In the proposed methods, to take advantage of orthogonality of Walsh-Hadamard codes, we assign additional bits to the each orthogonal code. For example, for Walsh-Hadamard codes matrix $\mathbf{C}$ with length $N_{p}=4$, we simply assign the information bits in each row vector as given in Table 1. For instance, if incoming bits for orthogonal Walsh-Hadamard code word is $\left[\begin{array}{ll}0 & 1\end{array}\right]$, we select $\mathbf{c}_{3}=\left[\begin{array}{llll}+1 & +1 & -1 & -1\end{array}\right]$ as a pilot symbols in the related OFDM symbol. These designs also can be extending for higher $N_{p}$ values. Proposed method is capable of enhancing the spectral efficiency without extra energy consumption compared to conventional OFDM by assigning extra bits to the index of orthogonal pilot symbols. As a result, the index of the Walsh-Hadamard codes convey an additional information and complete (realize) channel estimation. Moreover, proposed techniques do not need any prior knowledge at the receiver and extra energy consumption.

This paper is organized as follows. Section II demonstrates the principle of proposed schemes. Section III introduces the spectral efficiency of the proposed schemes. The receiver design and simulation results are given in Section IV and
Section V, respectively. Finally, conclusion is presented in Section VI.

\section{PROPOSED METHODS}

The general system block diagram is given in Fig.1. First, incoming $b$ bits are split into $b_{1}$ and $b_{2}$ bits in each OFDM symbol. Then, $b_{1}$ bits are mapped onto the $M$-ary signal constellation and the remaining $b_{2}$ bits are used to select a pilot signal from a predefined set of orthogonal pilot vectors. We proposed two methods which are inserted the selected pilot tones considering the whole OFDM and subgroups. In the first system, only one pilot signal is selected according to the index bits $\left(b_{2}\right)$, while in the second system, OFDM is divided into subgroups, and the pilot signal is selected according to the index bit for each subgroup. This allows more bits to be moved in an OFDM symbol. The proposed schemes are explained as follows:

\section{A) Orthogonal Pilot Code Index Modulation (OPC-IM):}

In the proposed Orthogonal Pilot Code Index Modulation (OPC-IM) scheme, $b_{2}=\left\lfloor\log _{2}\left(N_{p_{1}}\right)\right\rfloor$ bits are assigned to the index of orthogonal pilot tone for each OFDM symbol where $N_{p_{1}}$ is number of the pilot tone. Fig. 2 shows an illustrative frame structure of the proposed OPC-IM scheme where total number of subcarrier $N=32$, total data symbols $N_{d}=24$ and number of pilot tones $N_{p_{1}}=8$. For this parameter set, additionally $b_{2}=\left\lfloor\log _{2}(8)\right\rfloor=3$ bits are carried. For instance,

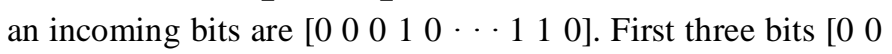


0] are mapped to orthogonal pilot code index, (i.e., the code $\mathbf{c}_{1}$ will be used for pilot symbols according to the Table II) then the remained bits $\left[\begin{array}{llllll}1 & 0 & \cdots & 1 & 1 & 0\end{array}\right]$ are used for mapping

TABLE I

\begin{tabular}{c|c}
\multicolumn{2}{c}{ A LOOK-UP TABLE FOR $N_{p}=4$} \\
Bits & Pilot Tone Codes \\
\hline$[0,0]$ & $c_{1}=[+1,+1,+1,+1]$ \\
\hline$[0,1]$ & $c_{2}=[+1,-1,+1,-1]$ \\
\hline$[1,0]$ & $c_{3}=[+1,+1,-1,-1]$ \\
\hline$[1,1]$ & $c_{4}=[+1,-1,-1,+1]$ \\
\hline
\end{tabular}

TABLE II

A LOOK-UP TABLE FOR $N_{p}=8$

\begin{tabular}{c|c} 
Bits & Pilot Tone Codes \\
\hline \hline$[0,0,0]$ & $c_{1}=[+1,+1,+1,+1,+1,+1,+1,+1]$ \\
\hline$[0,0,1]$ & $c_{2}=[+1,-1,+1,-1,+1,-1,+1,-1]$ \\
\hline$[0,1,0]$ & $c_{3}=[+1,+1,-1,-1,+1,+1,-1,-1]$ \\
\hline$[0,1,1]$ & $c_{4}=[+1,-1,-1,+1,+1,-1,-1,+1]$ \\
\hline$[1,0,0]$ & $c_{5}=[+1,+1,+1,+1,-1,-1,-1,-1]$ \\
\hline$[1,0,1]$ & $c_{6}=[+1,-1,+1,-1,-1,+1,-1,+1]$ \\
\hline$[1,1,0]$ & $c_{7}=[+1,+1,-1,-1,-1,-1,+1,+1]$ \\
\hline$[1,1,1]$ & $c_{8}=[+1,-1,-1,+1,-1,+1,+1,-1]$ \\
\hline
\end{tabular}

according to $M$-ary modulation scheme. Therefore, in the proposed OPC-IM, in contrast to classical OFDM, pilot symbols are used to not only estimate the channel response, but also convey additional data bits through the index domain while maintaining same power consumption (with OFDM system with PSA-CE).

\section{B) Subblock Orthogonal Pilot Code Index Modulation (SOPC-IM):}

To increase the spectral efficiency of proposed OPC-IM method, an OFDM symbol is divided into subblocks with length $k=N / s$, where $s$ represents number of subblock and $N$ represents the length of the inverse fast Fourier transform (IFFT). Then, we perform OPC-IM method for each subblock. The process in all subblocks are independent and same of each other. An illustrative frame structure of the proposed Subblock Orthogonal Pilot Code Index Modulation (SOPC-IM) scheme where total number of subcarriers $N=$ 32 , total data symbols $N_{d}=24$, number of pilot tones for each subblock $N_{p_{2}}=4$ and number of subblock $s=2$ is given in Fig. 3. As seen from this figure, there are two subblocks and each of them use four orthogonal pilot tones for CE. For example, assume that an incoming bit sequence of is $\left[\begin{array}{lllllll}1 & 0 & 0 & 1 & 0\end{array}\right.$

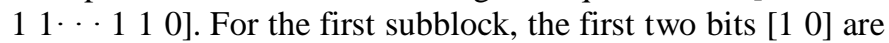
mapped to orthogonal pilot code index 3 (i.e., the code $\mathbf{c}_{3}$ will be used for pilot symbols in first subblock) and then incoming bits [0 1] are used for subblock 2 (i.e., the code $\mathbf{c}_{2}$ will be used for pilot symbols in second subblock) according to Table 1. Therefore, the proposed SOPC-IM method has more spectral efficiency than OPC-IM thanks to independent pilot tone selection for each subblock. Finally, remaining bits $\left[\begin{array}{llllllll}0 & 1 & 1 & \cdots & \cdots\end{array}\right.$ $10]$ are used for $M$-ary modulation scheme as usual.

\section{SPECTRAL EFFICIENCY}

The spectral efficiency of classical OFDM, without taking into account the cyclic prefix (CP) overhead, is given as follows:

$$
\eta_{\text {OFDM }}=\frac{b_{1}}{N}=\frac{\left(N-N_{p_{1}}\right) \log _{2} M}{N}
$$

It is clear that, pilot tones cause the loss of the spectral efficiency. The spectral efficiency of the proposed OPC-IM and SOPC-IM are given in (2) and (3), respectively.

$$
\begin{aligned}
& \eta_{\mathrm{OPC}-\mathrm{IM}}=\frac{b_{1}+b_{2}}{N}=\frac{\left(N-N_{p_{1}}\right) \log _{2} M+\log _{2}\left(N_{p_{1}}\right)}{N} \\
& \eta_{\text {SOPC-IM }}=\frac{b_{1}+b_{2}}{N}=\frac{\left(N-k N_{p_{2}}\right) \log _{2} M+k \log _{2}\left(N_{p_{2}}\right)}{N}
\end{aligned}
$$

Table III shows the spectral efficiency of the proposed methods for different modulation size. It is clear that the proposed SOPC-IM method has \%16.66 more spectral efficiency than classical OFDM system with BPSK modulation scheme.

TABLE III

SPECTRAL EFFICIENCIES FOR $N=256, N_{p_{1}}=64, N_{p_{2}}=4$ AND $k=16$.

\begin{tabular}{c|c|c|c} 
& OFDM & OPC-IM & SOPC-IM \\
\hline BPSK & 0.7500 & 0.7734 & 0.8750 \\
\hline QPSK & 1.500 & 1.5234 & 1.6250 \\
\hline 16-QAM & 3.000 & 3.0234 & 3.1250 \\
\hline
\end{tabular}

\section{RECEIVER DESIGN}

Let $\mathbf{x}_{F}$ be the transmitted OFDM symbol as follows:

$$
\mathbf{x}_{T}=\left[\mathbf{x}(1), \mathbf{x}(2), \cdots, \mathbf{c}_{i}(1), \cdots, \mathbf{x}(d), \mathbf{x}(d+1), \cdots, \mathbf{c}_{i}(p), \cdots, \mathbf{x}\left(N_{d}\right), \mathbf{c}_{i}\left(N_{p}\right)\right]
$$

where $d=1,2,3, \ldots, N_{d}, p=1,2,3, \ldots, N_{p}, \mathbf{x}$ is data symbol vector and $\mathbf{c}_{i}$ is selected Walsh-Hadamard code. After applying IFFT, the OFDM symbol given as:

$$
\mathbf{x}_{F}=\mathbf{W}_{N}^{H} \mathbf{x}_{T}
$$

where $\mathbf{W}_{N}$ is the FFT matrix with $\mathbf{W}_{N}^{H} \mathbf{W}_{N}=N \mathbf{I}_{N}$. Then, guard interval (or CP) is added to $\mathbf{x}_{F}$. The signal is converted from 
parallel to serial then the resulting OFDM symbol is transmitted through the channel. The vector of channel impulse response (CIR) coefficients are given as $\mathbf{h}=\left[h_{1}, h_{2}, \ldots, h_{N_{L}}\right]^{T}, \quad$ whose elements have complex Gaussian distribution as $C N\left(0,1 / N_{L}\right)$ where $N_{L}$ is the channel length. Hence, the received signal is given as

$$
y_{F}(f)=h_{F}(f)+w_{F}(f)
$$

where $w_{F}(f)$ and $h_{F}(f)$ are the noise samples and the channel fading coefficients in the frequency domain, respectively. codes as follows: The proposed methods are first used to detect the index of Walsh-Hadamard codes as follows:

$$
\frac{1}{N_{p}} \sum_{n=1}^{N_{p}} \mathbf{c}_{i}(n) \mathbf{c}_{j}(n)= \begin{cases}1, & i=j \\ 0, & i \neq j\end{cases}
$$

After determining the orthogonal pilot code $\mathbf{c}_{j}$, the frequency response of channel at the pilot positions can be estimated by using least square (LS) method as $\hat{h}\left(n_{c}\right)=y_{F}\left(n_{c}\right) / \mathbf{c}_{j}\left(n_{p}\right)$. Finally, cubic SPLINE interpolation technique is used in the process of constructing the whole channel response [6]. Then the first $b_{2}$ bits are demodulated according to the detection

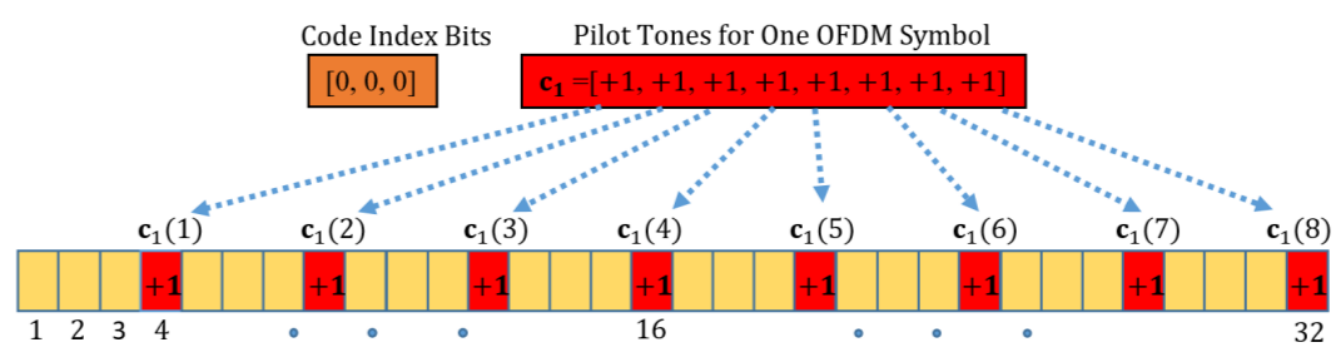

Data Symbol $\quad \square$ Incomming Bits for Orthogonal Pilot Symbols

Fig.2. An illustrative frame structure of the proposed OPC-IM scheme with $N=32, N_{d}=24$ and $N_{p_{1}}=8$.

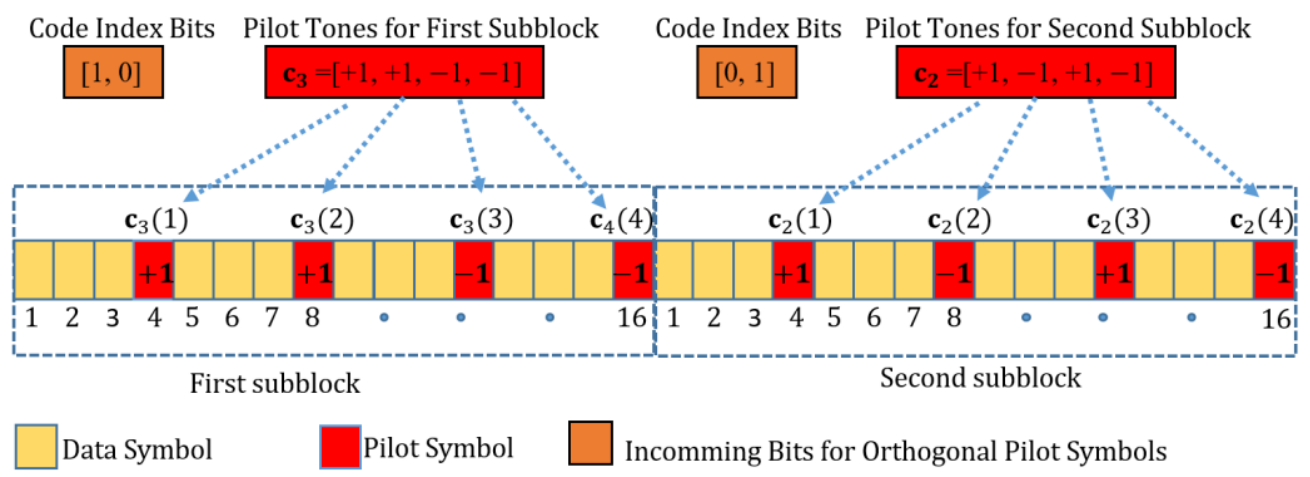

Fig.3. An illustrative frame structure of the proposed SOPC-IM scheme with $N=32, N_{d}=24$ and $N_{p_{2}}=4$ for each subblock.

Then, the received signals at pilot subcarriers can be expressed as follows:

$$
y_{F}\left(n_{c}\right)=\mathbf{c}_{i}\left(n_{p}\right) h_{F}\left(n_{c}\right)+w_{F}\left(n_{c}\right)
$$

where $n_{p}=1,2, \ldots, N_{p}, \quad n_{c}=N / N_{p}, 2 N / N_{p}, \ldots, N$, and $\mathbf{c}_{i}$ is the selected orthogonal pilot symbol according to index bits $b_{2}$. Than by correlating received signal (7) with WalshHadamard matrix $\mathbf{C}$ the orthogonal index of the orthogonal pilot code can be determined easily thanks to orthogonality of result in (7) using the look-up table for index of codes. To obtain the transmitted information $b_{1}$ bits on each OFDM symbols, the receiver uses hard decision decoding for $M$-ary symbols. As a result, proposed methods not only complete channel estimation without a loss of power, but also transmit additional information bits without extra bandwidth.

\section{Simulation Results}

Bit error rate (BER) performance of the proposed techniques are evaluated for different $N_{d}$ and $N_{p}$ values over 
frequency selective Rayleigh fading channels with length $N_{L}=$ 10. Signal to noise ratio (SNR) is described as $E_{b} / N_{0}$ where $N_{0}$ is the noise variance and $E_{b}$ is energy per bit. We assume that the receiver perfectly determines the index bits of WalshHadamard codes. The BERs of conventional OFDM and OPCIM are given in Fig. 4 as functions of the SNR for PSA channel estimation. As shown in from Fig. 4, the proposed OPC-IM method achieves almost same performance of the classical OFDM with different modulation scheme such as BPSK, QPSK, 8-QAM, 16-QAM and 64-QAM. Fig. 5 illustrates the BER of SOPC-IM and conventional OFDM as functions of the SNR. As shown that proposed SOPC-IM method achieves better performance than the conventional OFDM for lower modulation size. Because, at lower modulation size, the percentage of the bits which are carried by the index of orthogonal pilot tones are higher than that of the lower modulation size.

Table IV shows the BER gain of proposed systems compared to the classical pilot-aided OFDM system at a specific $E_{b} / N_{0}$ value. The BER gain $\left(\varsigma_{\varphi}\right)$ at $E_{b} / N_{0}=30 \mathrm{~dB}$ is calculated by using $\varsigma_{\varphi}=\frac{B E R_{\phi, 30 d B}-B E R_{\varphi, 30 d B}}{B E R_{\varphi, 30 d B}}$, where $\phi$ and $\varphi$ denote classical pilot-aided OFDM system and the proposed system ( $\varphi \in\{O P C-I M, S O P C-I M\})$, respectively. As can be seen from Table IV, BER performance of the proposed systems slightly outperforms the classical pilot-aided OFDM system. The performance gap between SOPC-IM system and the classical OFDM system is also seen from Fig. 5.

Fig. 6 depicts the effect of increasing the total number of the subcarriers $N$ on the spectral efficiency of the proposed techniques and conventional OFDM scheme. It is demonstrated that spectral efficiency for proposed methods are higher than conventional OFDM. For example, spectral efficiency for $N=1024$ and 8-QAM is $2.25 \mathrm{bpcu}$ for conventional OFDM while the proposed methods have $\eta_{\text {SOPC }}=2.29 \mathrm{bpcu}$ and $\eta_{\text {SOPC }}=2.37 \mathrm{bpcu}$ for OPC-IM and SOPC-IM respectively. Hence, proposed methods have higher spectral efficiency than conventional OFDM.

TABLE IV

BER GAIN OF THE PROPOSED SYSTEMS COMPARED TO THE CLASSICAL PILOT-AIDED OFDM SYSTEM AT $E_{B} / N_{0}=30 \mathrm{~dB}$.

\begin{tabular}{c|c|c} 
& $\begin{array}{c}\text { BER Gain of the } \\
\text { OPC-IM system at } \\
E_{b} / N_{0}=30 \mathrm{~dB}\left(1 \times 10^{-2}\right)\end{array}$ & $\begin{array}{c}\text { BER Gain of the } \\
\text { SOPC-IM system at } \\
E_{b} / N_{0}=30 \mathrm{~dB}\left(1 \times 10^{-2}\right)\end{array}$ \\
\hline \hline BPSK & 1.82 & 16.67 \\
\hline QPSK & 0.91 & 8.33 \\
\hline 8-QAM & 0.61 & 5.56 \\
\hline 16-QAM & 0.46 & 4.17 \\
\hline 64-QAM & 0.30 & 2.78 \\
\hline
\end{tabular}

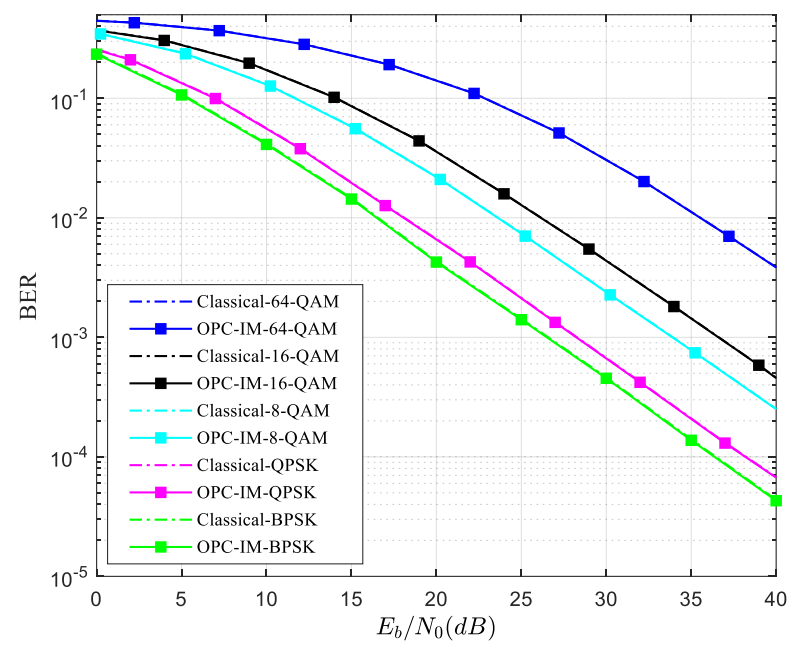

Fig.4. BER performances of proposed OPC-IM scheme and conventional OFDM with $N_{d}=512$, and $N_{p_{1}}=128$.

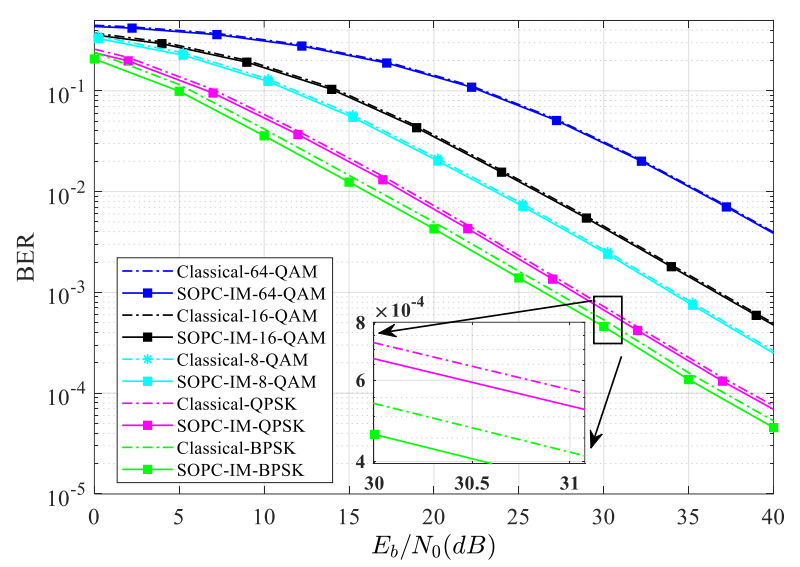

Fig.5. BER performances of proposed SOPC-IM scheme and conventional OFDM with $N_{d}=512, k=32$ and $N_{p_{2}}=4$ for each subblock.

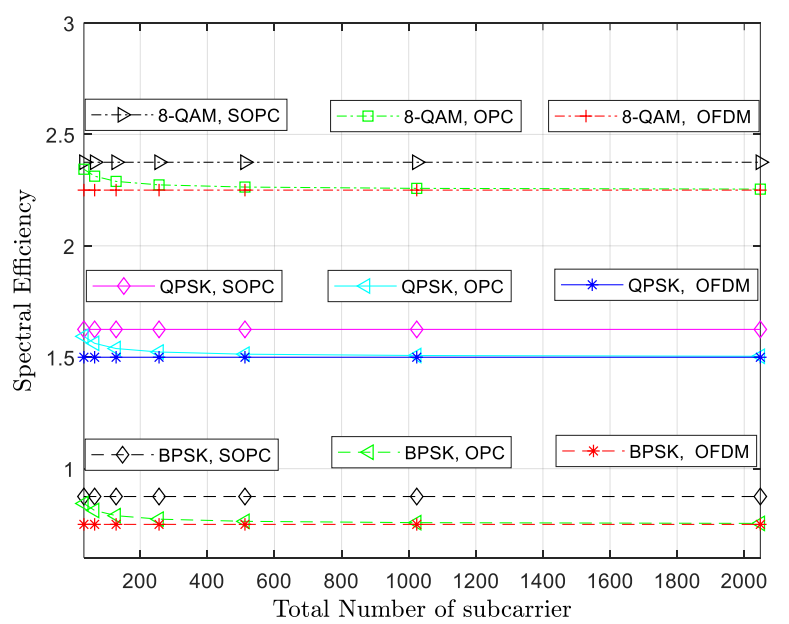

Fig.6. Spectral efficiency with varying $N$ values and modulation schemes. 


\section{CONCLUSIONS}

In this work, new pilot design approaches are proposed to enhance the spectral efficiency of conventional OFDM system. To obtain the high spectral efficiency, our designs use Walsh-Hadamard codes. Using these codes in each OFDM symbols shows that the proposed methods have higher spectral efficiency, better BER performance and no extra energy consumption with tolerable complexity compared to currently best known OFDM scheme.

\section{REFERENCES}

[1] R. Nee, P. Ramjee, "OFDM for wireless multimedia communications," Artech House, Inc., 2000.

[2] K. Fazel, S. Kaiser. Multi-carrier and spread spectrum systems: from OFDM and MC-CDMA to LTE and WiMAX. John Wiley \& Sons, 2008.

[3] M. Ergen, Mobile broadband: including WiMAX and LTE. Springer Science \& Business Media, 2009.

[4] B. Kamislioglu and A.Akbal, "Effect of doubly selective channels about bit error probability in OFDM and FBMC," European Journal of Technique, Vol. 7 No.2, 2017, pp. 96-101.

[5] M. C. Necker and G. L. Stuber, "Totally blind channel estimation for ofdm on fast varying mobile radio channels," IEEE Trans. Wireless Comm., Vol.3, No.5, 2004, pp. 1514-1525.

[6] Y. Acar, H . Dogan, E. Basar, and E. Panayirci, “'Interpolation based pilot aided channel estimation for stbc spatial modulation and performance analysis under imperfect CSI," IET Comm., Vol.10, No.14, 2016, pp. 1820-1828.

[7] Y. Acar, H. Dogan, and E. Panayirci, "Pilot symbol aided channel estimation for spatial modulation-OFDM systems and its performance analysis with different types of interpolations", Wireless Personal Communications, Vol.94, No.3, 2017, pp. 1387-1404.

[8] Y. Acar, S. Aldırmaz and E. Başar, "Channel estimation for OFDM-IM systems," Turkish Journal of Electrical Engineering I\& Computer Sciences, 2019, 27.3: 1908-1921.

[9] B. Özbek, R. Yllmaz . "The Adaptive Channel Estimation For STBCOFDM Systems". IU-Journal of Electrical \& Electronics Engineering 5 (2011): 1333-1340.

[10] B. Kamişlioğlu, A. AKBAL, "LSE Channel Estimation and Performance Analysis of OFDM Systems". Frrat University Turkish Journal of Science and Technology 12 (2017): 53-57

[11] Y. Liu, et al. "Channel estimation for OFDM. IEEE Communications Surveys $\backslash \&$ Tutorials, 2014, 16.4: 1891-1908.

[12] R. Mesleh, H. Haas, C.W. Ahn,, and S. Yun, "Spatial modulation-a new low complexity spectral efficiency enhancing technique", 2006 First International Conference on Communications and Networking, China (pp. 15). IEEE., October 2006.

[13] Y. Acar, "Subblock Aided OFDM with Index Modulation," Balkan Journal of Electrical and Computer Engineering, 7 (2) , 156-161, (2019). DOI: $10.17694 /$ bajece. 514324

[14] S. Aldırmaz, Y. Acar, and E. Basar. "Adaptive dual-mode OFDM with index modulation." Physical Communication 30 (2018): 15-25.

[15] Y. Acar, and T. Cooklev. "High performance OFDM with index modulation." Physical Communication 32 (2019): 192-199.
[16] E. Basar, M. Wen, R. Mesleh, M. Di Renzo, Y. Xiao, and H. Haas, "Index modulation techniques for next-generation wireless networks," IEEE Access, Vol.5, 2017, pp. 16693-16746.

[17] Q. Wang, G. Dou, X. He, R. Deng, J. Gao, "Novel OFDM System Using Data-Nulling Superimposed Pilots With Subcarrier Index Modulation", IEEE Communications Letters, Vol. 22, No.10, 2018, pp. 2164-2167.

[18] D. A. Bell, "Walsh functions and Hadamard matrixes," IET Electronics Letter, Vol.2, No.9, 1966, pp. 340-341.

\section{BIOGRAPHY}

YUSUF ACAR received the B.S.E. degree (with honors), M.S.E. degree and Ph.D. degrees in Electrical and Electronics

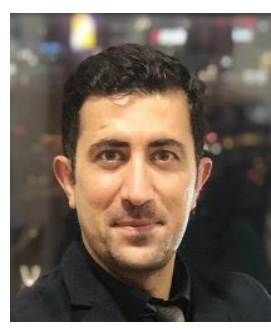
Engineering from Istanbul University, Istanbul, Turkey, in 2008, 2011 and 2015, respectively. In 2015, he joined the Department of Electrical and Electronics Engineering, Istanbul Kültür University as an Assistant Professor. He was a researcher at the Purdue University Fort Wayne (PFW), USA, between the September 2017 and September 2018. He is currently a senior system engineering at STM Savunma Teknolojileri Mühendislik ve Ticaret A.Ş., Ankara, Turkey. His general research interests cover communication theory, estimation theory, statistical signal processing, information theory and software-defined platforms. His current research activities are focused on radar signal processing.

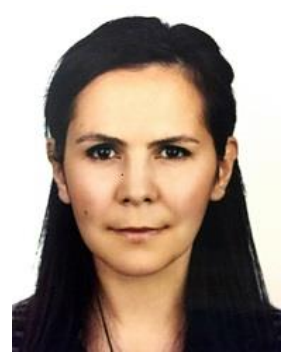

SULTAN ALDIRMAZ ÇOLAK received the B.S degree in Electronics and Communications Engineering from Kocaeli University, Kocaeli 2004 and the M.S. and PhD degrees in Yildiz Technical University (YTU), Istanbul 2006 and 2012, respectively. She was a visiting research scholar in the Department of Electrical and Computer Engineering of University of South Florida for the spring and summer of 2009. She is currently an Assistant Professor in the Electronics and Communications Engineering Department of Kocaeli University, Kocaeli, Turkey. Her research interests are in time-frequency signal processing, and communications theory. 